\section{Der Doktorandenpreis 2017 der Deutschen Lungenstiftung}

Im Rahmen des 58. Jahreskongresses der Deutschen Gesellschaft für Pneumologie und Beatmungsmedizin in Stuttgart wurde am 23. März 2017 der mit insgesamt 6000 Euro dotierte Doktorandenpreis der Deutschen Lungenstiftung verliehen. Der Preis für die beste klinische Arbeit mit dem Titel „Einfluss verschiedener, bei Lungentransplantation eingesetzter, immunsuppressiver Regime auf Regulatorische T-Zellen“ ging an Dr. Thuy Yen Vy Tran, für die beste experimentelle Arbeit zum Thema „BetaNikotinamid-Adenin-Dinukleotid als ein neuer Bronchodilator" an Dr. Innokentij Jurastow. Die Preisträger stellen nachfolgend die Inhalte ihrer Forschungsarbeit vor.

\section{Einfluss immunsuppressiver Regime auf Regulatorische T- Zellen}

Das angeborene und das erworbene Immunsystem sind für eine erfolgreiche Abwehr einer Vielzahl invadierender Pathogene von essenzieller Bedeutung. Eine große Gefahr des erworbenen Immunsystems ist die überschießende Immunreaktion gegen körpereigene oder harmlose fremde Strukturen wie inhalierte Substanzen oder Nahrungsbestandteile. Um Kollateralschäden der Entzündungsreaktion gering zu halten, müssen Immunreaktionen gegen Krankheitserreger limitiert werden. Diese wichtige Regulation des Immunsystems wird vor allem von den Regulatorischen T-Zellen ( $\left.T_{\text {regs }}\right)$ gewährleistet.

Transplantationen, als letzte Behandlungsmöglichkeit chronischer Erkrankungen, gehören heute zum klinischen Alltag. Ein Hauptproblem jeder Transplantation ist die Immunreaktion des Empfängerorganismus gegen das Transplantat. Das fremde Organ wird von den T-Zellen und den Antikörpern des Emp-

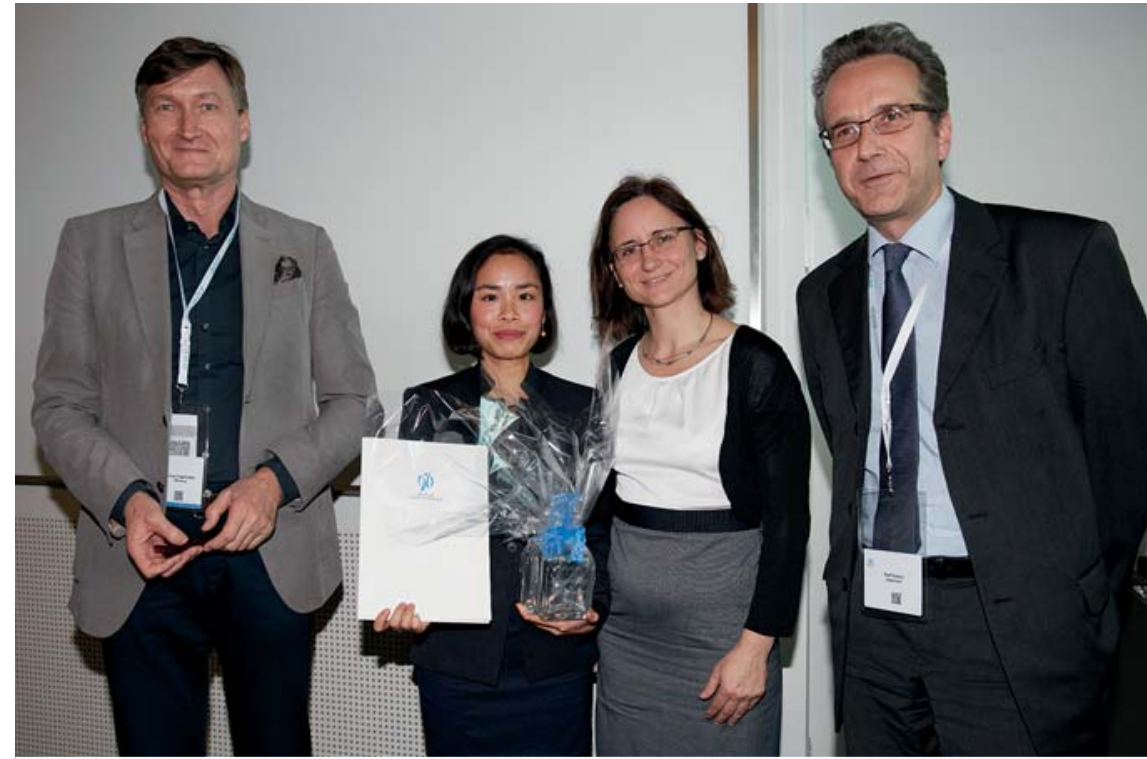

Von links: Prof. Dr. C. Vogelmeier, Vorsitzender der Deutschen Lungenstiftung e. V., Preisträgerin Dr. med. Thuy Yen Vy Tran, Boehringer Ingelheim Pharma GmbH \& Co KG vertreten durch Dr. Anke Kondla und Rolf Kaiser. Bildquelle: Mike Auerbach

fängers bekämpft, was eine Transplantatabstoßung zur Folge hat. Um die Abstoßung nach einer Lungentransplantation zu verhindern, existieren gegenwärtig verschiedene immunsuppressive Therapieregime. Welches Regime diese Aufgabe am Besten erfüllt, ist zurzeit nicht vollständig geklärt.

In dieser Dissertation wurde zum ersten Mal der Einfluss verschiedener immunsuppressiver Therapien auf die Kompartimente der Effektor-T-Zellen ( $\mathrm{T}_{\mathrm{effs}}$ ) und der CD4 + CD25 + Regulatorischen T-Zellen ( $T_{\text {regs }}$ ) bei Lungentransplantierten untersucht. Für die folgenden Untersuchungen wurde EDTA-Blut zur Generation von mononukleären Zellen des peripheren Blutes von 33 lungentransplantierten Patienten verwendet, die anhand der eingenommenen immunsuppressiven Therapien in drei Gruppen eingeteilt wurden. Mithilfe verschiedener immunologischer Methoden wie der Durchflusszytometrie, des CFSE- und Annexin-Assays zur Quantifizierung der Zellproliferation und der Apoptose sowie eines in vitro $\mathrm{T}_{\text {regs }}$-Suppression-Assays wurde der Einfluss verschiedener Immunsuppressiva (Tacrolimus, Ciclosporin, Rapamycin, Mycophenolat Mofetil) auf die Frequenz, den Phänotyp und die Funktion von $T_{\text {effs }}$ und $\mathrm{T}_{\text {regs }}$ untersucht.
Unter allen immunsuppressiven Therapien kam es zu einer signifikanten Reduktion des $\mathrm{T}_{\text {effs }}$-Kompartiments sowie zu einer Verschiebung des T-Helferzelle $\left(T_{H}\right)$-Gleichgewichts zugunsten der $T_{H} 2$, die sich in der verminderten Expression von IFN-y und Tim3 sowie der gesteigerten Expression von IL-4 und BTLA darstellte.

Im Gegensatz zur Reduktion des $\mathrm{T}_{\text {effs }}$ Kompartiments führte die Therapie mit Rapamycin (RPM), nicht aber die mit Calcineurininhibitoren, zu einer Vergrößerung des $\mathrm{T}_{\text {regs }}$-Kompartiments. In vitroUntersuchungen legten nahe, dass diese Vergrößerung durch die gesteigerte Proliferation, durch die de-novo-Generation der $T_{\text {regs }}$ sowie durch die verminderte Apoptose der $T_{\text {regs }}$ unter Einfluss von RPM bedingt ist. Des Weiteren führte die Gabe von RPM in vitro zu einer gesteigerten Kapazität der $\mathrm{T}_{\text {regs }}$ und somit zur Suppression von aktivierten $\mathrm{T}_{\text {effs }}$, die mit einer Hochregulation des Oberflächenmarkers CD62 $\mathrm{L}$ auf den $\mathrm{T}_{\text {regs }}$ assoziiert war.

Zusammenfassend zeigt diese Arbeit, dass die Therapie mit RPM im Vergleich zur Therapie mit Calcineurininhibitoren zu einer quantitativen und qualitativen Stärkung des $T_{\text {regs }}$-Kompartiments führt. 
Zukünftige Studien sollten daher untersuchen, ob der Einsatz von RPM bei lungentransplantierten Patienten zu einer Verbesserung des Transplantats- und Gesamtüberlebens führt.

Dr. Thuy Yen Vy Tran

\section{Beta-Nikotinamid-Adenin- Dinukleotid als ein neuer Bronchodilator}

Die Bronchokonstriktion oder die generelle Obstruktion der Atemwege hat eine große Bedeutung bei Erkrankungen des Menschen. Die wichtigsten Beispiele obstruktiver Atemwegserkrankungen stellen das Asthma bronchiale und die chronisch obstruktive Lungenerkrankung (COPD) dar.

Asthma bronchiale gehört zu den chronisch entzündlichen Atemwegserkrankungen und ist unter anderem durch eine bronchiale Hyperreaktivität und anfallartigen Husten mit teilweise vorkommendem Auswurf von zähem Schleim charakterisiert. Die Entzündungsreaktion der Bronchialschleimhaut spielt eine wichtige Rolle bei der Entstehung von Asthma. Hierbei spielen allergenspezifische $\mathrm{T}_{\mathrm{H}} 2$-Lymphozyten eine wichtige Rolle. Diese produzieren verschiedene Zytokine und Hormone und führen zu einer Rekrutierung von inflammatorisch wirkenden Zellpopulationen, was unter anderem zu einer bronchialen Obstruktion durch Bronchospasmus und zu einer Schleimhautschwellung durch eine entzündliche Infiltration führt. Die hinzukommende erhöhte Schleimbildung führt zu einer Verlegung der Atemwege und zu dem Gefühl der akuten Atemnot.

COPD ist eine chronisch obstruktive Lungenerkrankung, die sich im fortgeschrittenen Stadium zu einer Systemerkrankung erweitern kann und im Gegensatz zu Asthma bronchiale durch eine nicht komplett reversible Atemwegsobstruktion, vor allem durch den Bronchialschleim und durch Krampfzustände der Bronchialmuskulatur, gekennzeichnet wird. Die chronisch obstruktive Bron-

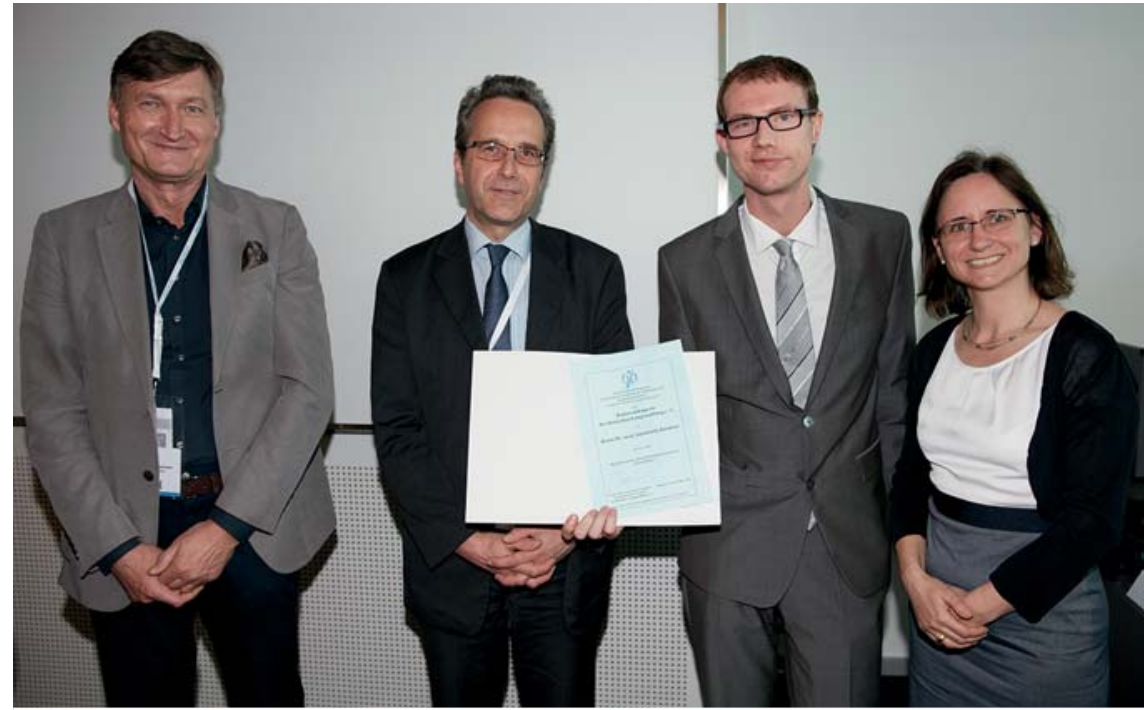

Von links: Prof. Dr. C. Vogelmeier, Vorsitzender der Deutschen Lungenstiftung e. V., Rolf Kaiser, Boehringer Ingelheim Pharma GmbH \& Co. KG, Preisträger Dr. med. Innokentij Jurastow und Frau Dr. Anke Kondla, Boehringer Ingelheim Pharma GmbH \& Co. KG. Bildquelle: Mike Auerbach

chitis und das Lungenemphysem werden mit in die COPD eingeschlossen.

Weltweit sind ungefähr 600 Mio. Menschen an COPD erkrankt, dabei stellt diese Erkrankung die vierthäufigste Todesursache dar. Leitsymptome bei dieser Krankheit sind Husten, Auswurf und Atemnot, dieses insbesondere bei Belastung. Die Hauptursache der COPD ist das Tabakrauchen. Der Entzündungsprozess bei COPD entsteht durch ein Ungleichgewicht der proinflammatorischen und antiinflammatorischen Zytokine. Diese Entzündungsreaktion führt zu systemischen Erkrankungen wie Kachexie, Depression, Osteoporose und auch zu einer deutlich erhöhten kardiovaskulären Morbidität mit Komplikationen wie Schlaganfall oder koronarer Herzerkrankung.

Die Therapie dieser zwei Erkrankungen richtet sich immer nach dem Schweregrad und ist durch die jeweils komplexe Ätiologie bis heute nicht kurativ. So beinhaltet die Therapie gegen Asthma hauptsächlich eine anti-inflammatorische Komponente durch Kortikosteroide und eine bronchorelaxierende Komponente durch kurz- oder langwirksame $\beta$ Adrenozeptoragonisten.
Das Therapiekonzept der COPD besteht aus kurzwirksamen und langwirksamen Bronchodilatatoren, Antimuskarinika und inhalativen Steroiden. Weiterhin kann systemischen Symptomen entgegengewirkt werden, zum Beispiel durch eine Ernährungstherapie zur Vermeidung der Kachexie und Lungensport.

Da $\beta$-NADin der Literatur als möglicher Neurotransmitter beschrieben wurde, welcher die glatte Muskulatur im menschlichen Intestinaltrakt relaxiert, wurde in dieser Arbeit untersucht, ob es auch in Atemwegen der Maus und des Menschen relaxierend wirkt und, falls ja, über welchen Signalweg diese Relaxation vermittelt wird. Atemwegskontraktion wurde in Organbadexperimenten (Maus, Mensch) und videomorphometrisch in lebenden Lungenschnittpräparationen (precision-cut lung slices $=$ PCLS) der Maus gemessen. Mittels einer in dieser Arbeit neu etablierten Methode konnten in Lungenschnitten parallel zur Bronchusfläche gleichzeitig Veränderungen der $\left[\mathrm{Ca}^{2+}\right]_{i}$ und der Membranpotenziale bestimmt werden. An kultivierten glatten Muskelzellen wurden Änderungen der $\left[\mathrm{Ca}^{2+}\right]_{i}$ und, durch FRET-Experimente, der $[\mathrm{cAMP}]_{i}$ nach Gabe von $\beta$-NAD untersucht. 
$\beta$-NAD relaxiert vorkontrahierte Atemwege sowohl der Maus als auch des Menschen. Dieser Effekt war vergleichbar mit der Relaxation nach Gabe von Salbutamol, einem $\beta_{2}$-Adrenozeptoragonisten. Im Gegensatz zu der Relaxation im Intestinaltrakt, welche durch purinerge Rezeptoren vermittelt wurde, war der relaxierende Effekt durch Antagonisten purinerger Rezeptoren nicht hemmbar. AuBerdem war diese Relaxation unabhängig von der Hemmung G-Protein-gekoppelter Rezeptoren und vieler anderer Blocker der Dilation glatter Muskelzellen. In kultivierten Zellen und in glatten Muskelzellen der Bronchi in PCLS konnte eine $\mathrm{Er}$ höhung der $\left[\mathrm{Ca}^{2+}\right]_{i}$ beobachtet werden, welche simultan mit einer Depolarisation und einer Relaxation einherging. Da von den eingesetzten Blockern einzig bei Rolipram, einem Blocker der PDE-4, eine Veränderung in der Relaxationskinetik, nämlich eine Verstärkung, beobachtet wurde, wurde cAMP mittels FRET (Förster-Resonanzenergietransfer) gemessen. Hier zeigte sich eine $\beta$-NAD-induzierte $\mathrm{Er}$ höhung der $[\mathrm{cAMP}]_{\mathrm{i}}$, welche durch einen Blocker der Iöslichen Adenylatzyklase (AC) gehemmt werden konnte, der auch in Organbadversuchen die $\beta$-NAD-induzierte Relaxation signifikant hemmte.

Diese Ergebnisse zeigen $\beta$-NAD als einen neuen Bronchodilator, welcher durch Aktivierung der löslichen AC und durch die Erhöhung der [CAMP $]_{i}$ zu einer Relaxation glatter Muskelzellen führt. Durch die hier gezeigte relaxierende Wirkung an glatten Muskelzellen der Atemwege und die in anderen Arbeiten gezeigte antiinflammatorische Komponente stellt $\beta$-NAD möglicherweise ein neues Therapeutikum bei bronchokonstriktiven Erkrankungen dar.

Dr. Innokentij Jurastow 